\section{Electron drift velocity in hydrogen, nitrogen, oxygen, carbon monoxide, carbon dioxide and air at moderate $\mathrm{E} / \mathrm{N}$}

To cite this article: W Roznerski and K Leja 1984 J. Phys. D: Appl. Phys. 17279

View the article online for updates and enhancements.
Related content

- The ratio of lateral diffusion coefficient to
$\frac{\text { mobility for electrons in hydrogen and }}{\text { nitrogen at moderate E/N }}$
W Roznerski and K Leja
- The characteristic energy and ratio of
$\frac{\text { longitudinal diffusion coefficient to mobility }}{\text { for electrons in hydrogen and nitrogen }}$
W Roznerski, J Mechlinska-Drewko and K
Leja
- Drift velocity and longitudinal diffusion
$\frac{\text { coefficient of electrons in nitrogen and }}{\text { carbon monoxide }}$

Recent citations

$\frac{\text { Time evolution of vibrational temperatures }}{\text { in a } \mathrm{CO}_{2} \text { glow discharge measured with }}$
$\frac{\text { infrared absorption spectroscopy }}{\mathrm{B} \mathrm{L} \mathrm{M} \mathrm{Klarenaar} \mathrm{et} \mathrm{al}}$
$\frac{\text { Measurement of ionization, attachment. }}{\text { detachment and charge transfer rate }}$
$\frac{\text { coefficients in dry air around the critical }}{\text { electric field }}$
A Hösl et al
- Electron scattering cross sections for the
$\frac{\text { modelling of oxvgen-containing plasmas }}{\text { Luís Lemos Alves et al }}$ 


\title{
Electron drift velocity in hydrogen, nitrogen, oxygen, carbon monoxide, carbon dioxide and air at moderate $E / N$
}

\author{
W Roznerski and K Leja \\ Institute of Physics. Technical University of Gdańsk, 80-952 Gdańsk. Poland
}

Received 22 August 1983

\begin{abstract}
The drift velocity of electrons in hydrogen. nitrogen. oxygen. carbon monoxide. carbon dioxide. and air free from water vapour and carbon dioxide. at ambient temperatures (293-296 K) has been measured by the modified Bradbury-Nielsen time-of-flight method over the range of the reduced electric field $E / N: 0.05 \leqslant E / N \leqslant 250 \mathrm{Td} .0 .1 \leqslant E / N \leqslant 250 \mathrm{Td}$. $1.0 \leqslant E / N \leqslant 275 \mathrm{Td} .0 .1 \leqslant E / N \leqslant 150 \mathrm{Td} .0 .6 \leqslant E / N \leqslant 250 \mathrm{Td}$ and $0.05 \leqslant E / N \leqslant 400 \mathrm{Td}$ respectively. The present results are compared with those obtained by other investigators.
\end{abstract}

\section{Introduction}

It is well known that knowledge of electron transport coefficients in gases is necessary to determine the appropriate cross-sections for elastic and inelastic processes in the collisions of low energy electrons with the molecules of the medium. The drift velocity is one of these transport coefficients. The traditional methods of measuring transport coefficients are based on the analysis of the spatial and temporal distribution of the electron swarm, diffusing through a gas in a homogeneous electric field in the space between cathode and anode. This can lead to significant experimental errors due to boundary effects. Attempts to minimise these effects are fully justified and useful (Breare and von Engel 1964. Blevin et al 1976a). The quantitative estimation of the errors caused by the boundary effects resulting from large density gradients near the electrode surfaces (Lowke et al 1977, Braglia and Lowke 1979) is very difficult under actual experimental conditions. Comparison of the consistent experimental data obtained by a traditional measurement method with those obtained by the method substantially minimising the boundary effects could facilitate the estimation of these effects, particularly at elevated values of reduced electric field.

From the earlier analyses of drift velocity measurements it follows that the Bradbury-Nieisen time-of-flight method gives, under the appropriate experimental conditions, highly precise results (Crompton et al 1967). The measurements carried out using the system suggested by Blevin and Hasan (1967) have shown that this method makes it possible to obtain advantageous data over a relatively wide range of $E / N$ values at a slight reduction of the measurement accuracy.

The aim of this work has been to obtain consistent drift velocity data, within the method applied and the experimental errors, for pure molecular gases: hydrogen, 
nitrogen, oxygen, carbon monoxide, carbon dioxide and dry air at moderate $E / N$ values using the Blevin and Hasan (1967) technique.

\section{Experimental method}

In the experiment of Blevin and Hasan (1967) the usual Bradbury-Nielsen type of coplanar grid system was replaced by a double grid system (figure 1) with the electrical shutters operated by an AC voltage applied between the adjacent grids. In the present version of the experimental system the grid separation was about $0.5 \%$ of the drift

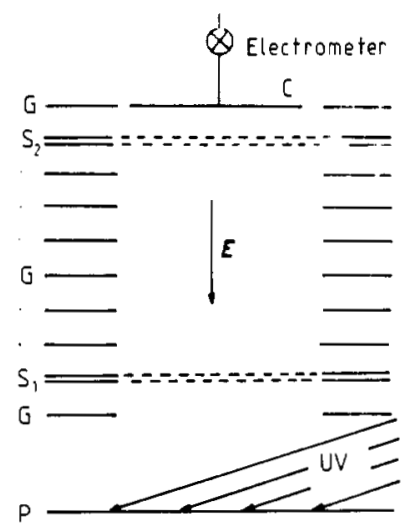

Figure 1. Schematic diagram of apparatus used in the present experiment. Key: P, photocathode; $\mathrm{G}$, guard rings; $S$, electrical shutters; $\mathrm{C}$, collector.

distance (which was $10.97 \mathrm{~cm}$ ). For the measurements a HF power generator controlled by a digital timer was used, with an amplitude of $0-21 \mathrm{~V}$ over the applied range of frequency from $20 \mathrm{kHz}$ to $20 \mathrm{MHz}$. The DC voltage applied to the electrodes of the drift tube changed from 20 to $500 \mathrm{~V}$ and was known to an accuracy of $0.01 \mathrm{~V}$ in the range 20-120 V and to an accuracy of $0.1 \mathrm{~V}$ above $120 \mathrm{~V}$. The source of electrons was a goldleaf photocathode illuminated by UV radiation. The rate of increase in pressure for the system isolated from a liquid nitrogen cooling trap was less than $10^{-8}$ Torr litre $\mathrm{s}^{-1}$. The measurements of the gas pressure were made with three mercury-filled McLeod gauges, connected with the liquid nitrogen or the solid carbon dioxide cooling traps, which covered the range of pressure 0.097-39.76 Torr. All measurements were carried out at room temperature, i.e. $294.5 \pm 1.5 \mathrm{~K}$, and temperature variation was controlled with an accuracy of $0.5 \mathrm{~K}$. For the measurements spectrally pure molecular gases (purity higher than $99.99 \%)$ and dry air $\left(\mathrm{H}_{2} \mathrm{O}<10 \mathrm{PPM}\right.$ and $\left.\mathrm{CO}_{2}<1 \mathrm{PPM}\right)$ have been used.

\section{Results and discussion}

For all gases studied in this work, except oxygen for $E / N \leqslant 2.5 \mathrm{Td}$, the experimental error has been estimated as $\pm 1.5 \%$ for $E / N \leqslant 50 \mathrm{Td}$, and $\pm 3 \%$ for the values above $50 \mathrm{Td}$. In the case of oxygen the error for $E / N \leqslant 2.5 \mathrm{Td}$ has been taken to be $\pm 5 \%$, for $2.5<E / N \leqslant 50 \mathrm{Td}$ as $\pm 2 \%$, and for $E / N>50 \mathrm{Td}$ as $\pm 3 \%$. The percentage differences 


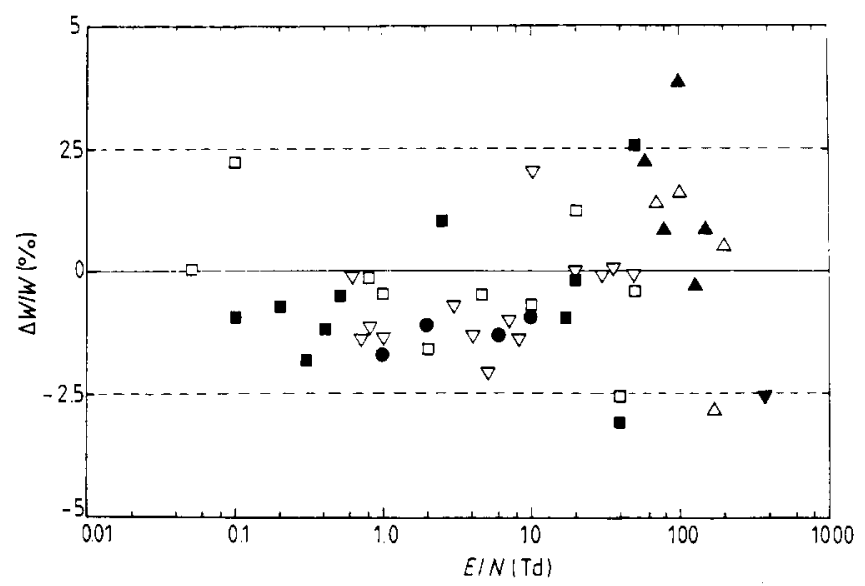

Figure 2. Percentage difference between the present results and those of other investigators for hydrogen, nitrogen, and carbon dioxide. Hydrogen: $\mathbf{0}$ (Lowke 1963); (Robertson 1971); $\Delta$ (Blevin and Hasan 1967); $\mathbf{\nabla}$ (Saelee 1976). Nitrogen: $\square$ (Lowke 1963); $\triangle$ (Blevin and Hasan 1967). Carbon dioxide: $\nabla$ (Elford and Haddad 1980).

between the present results and those of other investigators for hydrogen, nitrogen and carbon dioxide (whose estimated experimental errors did not exceed $\pm 4 \%$ over the $E / N$ range up to about $250 \mathrm{Td}$ ) are shown in figure 2 . These differences, except for a few individual points, lie within the limits of error of the present experiments, while for all experimental points they lie within the limits of combined errors of the experiments which are compared.

Table 1 contains the results of the drift velocity measurements for $E / N \geqslant 50 \mathrm{Td}$ obtained in the present experiment.

\subsection{Hydrogen}

The comparison between our results and those of Lowke (1963), Robertson (1971), Blevin and Hasan (1967) and Saelee (1976) is shown in figure 2. The majority of our

Table 1. Drift velocity of electrons in hydrogen, nitrogen, oxygen, carbon monoxide, carbon dioxide and air free from water vapour and carbon dioxide at 293-296 K.

\begin{tabular}{rllllll}
\hline & \multicolumn{7}{c}{ Drift velocity $W\left(10^{4} \mathrm{~m} \mathrm{~s}^{-1}\right)$} \\
\cline { 2 - 7 }$E / N$ & & & $\begin{array}{l}\text { Carbon } \\
\text { (Td) }\end{array}$ & Hydrogen & Nitrogen & Carbon \\
dioxide & Air \\
\hline 50 & 5.861 & 6.065 & 9.476 & 4.534 & 10.96 & 8.725 \\
75 & 9.244 & 8.533 & 12.11 & - & - & 11.82 \\
100 & 13.35 & 10.68 & 14.93 & 8.360 & 12.53 & 13.24 \\
125 & 17.27 & - & 16.81 & - & 13.32 & 14.12 \\
150 & 21.48 & 15.10 & 19.39 & 12.91 & 14.01 & 16.32 \\
175 & 25.42 & 18.15 & 23.24 & - & 15.55 & 18.99 \\
200 & 30.45 & 21.31 & 25.93 & - & 17.37 & 21.52 \\
250 & 40.09 & 27.60 & 30.08 & - & 21.01 & 25.71 \\
300 & - & - & - & - & - & 31.65 \\
400 & - & - & - & - & - & 39.60 \\
\hline
\end{tabular}


data points agree within our experimental errors with the results of the references given. Our remaining results lie within the limits of the combined errors of our experiment and those of the references. The additional point measured for $E / N=367.2 \mathrm{Td}$ confirms the good agreement between our results and those of Saelee (1976). Except for the results for $E / N=55 \mathrm{Td}$, our results agree fairly well with the data of Jaeger and Otto (1962) and with the results of Frommhold (1960), for $E / N \leqslant 120 \mathrm{Td}$. Larger discrepancies with the data of Frommhold (1960) are found over the $E / N$ range $120 \leqslant E / N \leqslant 250 \mathrm{Td}$, where all our data points lie between 6 and $17 \%$ higher. Good agreement was found with the results of Blevin et al (1976b), obtained from observations of the photon flux produced in a Townsend discharge, but our data are lower than those obtained by Blevin et al (1978) using the Monte Carlo simulation technique and the difference between both sets of data increases to about $10 \%$ at $200 \mathrm{Td}$.

\subsection{Nitrogen}

Throughout the whole range of $E / N$ values good agreement (within the combined errors of each two sets of experimental data) has been observed with the results of Lowke (1963) and of Blevin and Hasan (1967) (figure 2). However, the discrepancies with the results of the latter work for both sets of data over the $E / N$ range $140 \leqslant E / N \leqslant 170 \mathrm{Td}$, increase from 3 to $6 \%$. A better agreement was found, over the same range of $E / N$, with the results of Tholl (1964). For the remaining values from the common $E / N$ range our results agree with those of Tholl (1964) within the experimental error of our work. For $E / N \leqslant 120$ Td our measured results are in very good agreement with the data of Prasad and Smeaton (1967). Over the $E / N$ range $150 \leqslant E / N \leqslant 250 \mathrm{Td}$ our data points are found to be higher than the corresponding results of Wagner (1964), and the discrepancies increase monotonically from about $1 \%$ for $150 \mathrm{Td}$ to about $15 \%$ for $250 \mathrm{Td}$. From

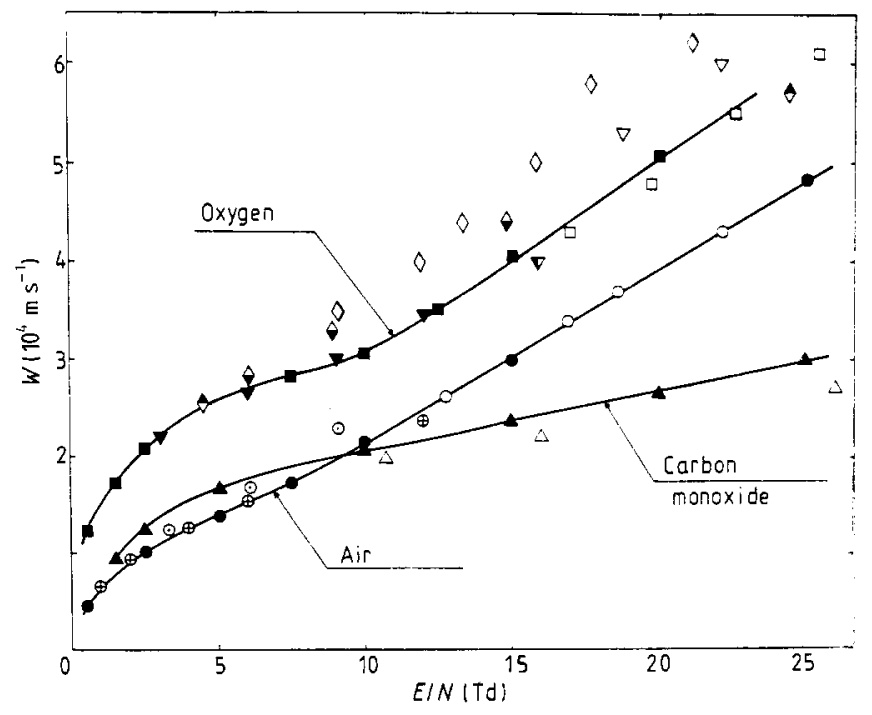

Figure 3. Drift velocities in oxygen, carbon monoxide and air below $27.5 \mathrm{Td}$. Oxygen: $\boldsymbol{\nabla}$ (Crompton and Elford 1973); $\diamond$ (Doehring 1952); $\diamond$ (Fleming et al 1972); $\square$ (Herreng 1952); $\nabla$ (Naidu and Prasad 1970); $\nabla$ (Nielsen and Bradbury 1937); $\diamond$ (Pack and Phelps 1966); (present results). Carbon monoxide: $\triangle$ (Pack and Phelps 1962); $\boldsymbol{\Delta}$ (present results). Air: $\odot$ (Hessenauer 1967); $\bigcirc$ (Heylen 1962); $\oplus$ (Rees 1973); $\bigcirc$ (present results). 
comparison with the results of Fletcher and Reid (1980) it is seen that the agreement between both sets of data is good for $E / N<200 \mathrm{Td}$, while for $E / N \geqslant 200 \mathrm{Td}$ our results are found to be higher by about $7 \%$ at $200 \mathrm{Td}$ and about $10 \%$ at $250 \mathrm{Td}$. Some discrepancies have been noted in relation to the theoretical data of Pitchford and Phelps (1982). It is seen that the agreement is quite good at $100 \mathrm{Td}$ for their Monte Carlo simulation value, while for the remaining three $E / N$ values $(40,70$ and $200 \mathrm{Td}$ ) the discrepancies exceed the experimental errors of the present experiment independent of the drift velocity and calculation technique applied, and the maximum difference reaches about $12 \%$ at $200 \mathrm{Td}$. Good agreement was observed with the resuits of Monte Carlo simulations by Braglia et al (1982) for $100 \mathrm{Td}$ and Novak and Frechette (1983) for $200 \mathrm{Td}$. For 40 and $100 \mathrm{Td}$ discrepancies were found between our results and those presented in both quoted works which exceeded our experimental errors.

\subsection{Oxygen}

For the lowest $E / N$ values measured in this work we found agreement with the results of Reid and Crompton (1980) within the combined errors of both sets of the experimental data. Below 12 Td our results agree well with the data of Crompton and Elford (1973). We also found a fair agreement with the results of Fleming et al (1972) over the common $E / N$ range and with the data by Herreng (1952) for $E / N \leqslant 25 \mathrm{Td}$. The discrepancies observed with the data of other investigators for $E / N \leqslant 27.5 \mathrm{Td}$ are shown in figure 3 . For $20 \leqslant E / N \leqslant 100 \mathrm{Td}$ results of this work agree well with those of Naidu and Prasad (1970), but for $E / N \geqslant 100 \mathrm{Td}$ our data points are situated about $5 \%$ lower than the resuits of Naidu and Prasad (1970). There were larger discrepancies with the results of Frommhold (1964) over the $E / N$ range $100 \leqslant E / N \leqslant 275 \mathrm{Td}$ but the difference between both data sets decreases from about $20 \%$ at $100 \mathrm{Td}$ to about $3 \%$ at $275 \mathrm{Td}$. The two experimental points at 200 and $250 \mathrm{Td}$ measured by Schlumbohm (1965) lie about $5 \%$ higher than our's.

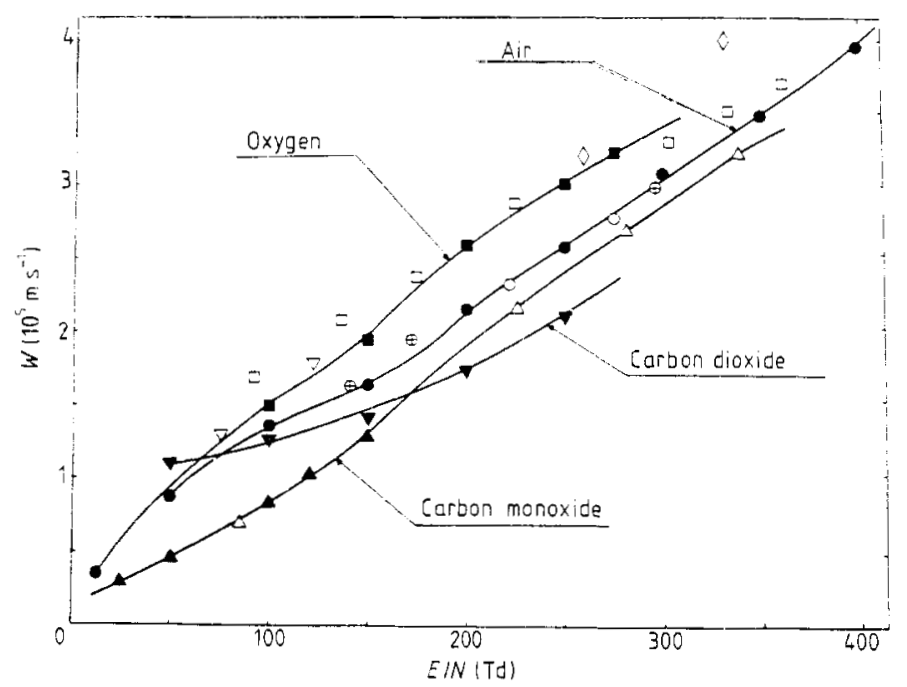

Figure 4. Drift velocities in oxygen, carbon monoxide, carbon dioxide and air at moderate $E / N$. Oxygen: $\square($ Frommhold 1964); $\nabla$ (Naidu and Prasad 1970); $\diamond($ Schlumbohm 1965); - (present results). Carbon monoxide: $\Delta$ (Saelee 1976); $\Delta$ (present results). Carbon dioxide: $\nabla$ (present results). Air: $\bigcirc$ (Frommhold 1974); $\oplus$ (Ryżko 1965): O (present results). 


\subsection{Carbon monoxide}

The agreement between the present results and those measured by Pack et al (1962) is good for $E / N \leqslant 0.8 \mathrm{Td}$, but over the $E / N$ range of $0.8 \leqslant E / N \leqslant 3$ Td our results are lower by rather more than $10 \%$. Above $10 \mathrm{Td}$ the results of this work are higher than the data of Pack et al (1962) and the difference between both sets of data increases up to 7\% at 25 Td (figure 3). Good agreement with the data calculated by Lowke and Parker (1969) is observed over the common $E / N$ range. Above $50 \mathrm{Td}$ our results agree well with the data of Saelee (1976).

\subsection{Carbon dioxide}

Below $50 \mathrm{Td}$ very good agreement (except for one point within about 1.5\%) was observed with the experimental results of Elford and Haddad (1980) (figure 2). Over the $E / N$ range $115 \leqslant E / N \leqslant 160 \mathrm{Td}$ our data points lie lower by about $15 \%$ than those measured by Frommhold (1960) and Schlumbohm (1965). Better agreement is seen with the result of Lowke and Parker (1969) at about $180 \mathrm{Td}$, where the difference is $7 \%$.

\subsection{Air}

For $E / N \leqslant 12$ Td the results of this experiment agree very well with those of Rees (1973), whereas the difference between our results and the results of Hessenauer (1967) increases up to about $18 \%$ at $9 \mathrm{Td}$. Our present results for $E / N \leqslant 50 \mathrm{Td}$ are in good agreement with the theoretical data of Heylen (1962). Some discrepancies are observed above $50 \mathrm{Td}$ within the limits 5 to $7 \%$, and all our data points, except for one point at about $100 \mathrm{Td}$, lie higher than those of Heylen (1962). Over the common $E / N$ range (up to about $300 \mathrm{Td}$ ) the present results agree well with the experimental data of Ryżko (1965).

\section{Acknowledgments}

The authors would like to thank Dr J P Novak for forwarding the data used in the preparation of this paper. This work was supported in part by the Institute of Experimental Physics of Warsaw University.

\section{References}

Blevin H A, Fletcher J and Hunter S R 1976b J. Phys. D: Appl. Phys. 91671

- 1978 Aust. J. Phys. 31299

Blevin H A, Fletcher J and Marzec L M 1976a J. Phys. D: Appl. Phys. 9465

Blevin H A and Hasan M Z 1967 A ust. J. Phys. 20735

Braglia G L and Lowke J J 1979 J. Phys. D: Appl. Phys. 121831

Braglia G L, Romano L and Diligenti M 1982 Phys. Rev. A 263689

Breare J M and von Engel A 1964 Proc. R. Soc. A 282390

Crompton R W and Elford M T 1973 Aust. J. Phys. 26771

Crompton R W, Elford M T and Jory R L 1967 A ust. J. Phys. 20369

Doehring A 1952 Z. Naturf. 7a 253

Elford M T and Haddad G N 1980 Aust. J. Phys. 33517

Fleming J A, Gray D R and Rees J A 1972 J. Phys. D: Appl. Phys. 5291 
Fletcher J and Reid I D 1980 J. Phys. D: Appl. Phys. 141685

Frommhold L 1960 Z. Phys. 160554

1964 Fortschr. Phys. 12597

Herreng P 1952 Cah. Phys. 387

Hessenauer H 1967 Z. Phys. 204142

Heylen A E D 1962 Proc. Phys. Soc. 79284

Jaeger $\mathrm{H}$ and Otto 1962 Z. Phys. 169517

Lowke J J 1963 Aust. J. Phys. 16115

Lowke J J and Parker J H 1969 Phys. Rev. 181302

Lowke J J, Parker J H and Hall C A 1977 Phys. Rev. A 151237

Naidu M S and Prasad A N 1970 J. Phys. D: Appl. Phys. 3957

Nielsen R A and Bradbury N E 1937 Phys. Rev. 5169

Novak J P and Frechette M 1983 J. Appl. Phys. in press

Pack J L and Phelps A V 1966 J. Chem. Phys. 441970

Pack J L, Voshall R E and Phelps A V 1962 Phys. Rev. 1272084

Pitchford L C and Phelps A V 1982 Phys. Rev. A 25540

Prasad A N and Smeaton G P 1967 Br. J. Appl. Phys. 18371

Rees J A 1973 Aust. J. Phys. 26427

Reid I D and Crompton R W 1980 Aust. J. Phys. 33215

Robertson A G 1971 Aust. J. Phys. 24445

Ryżko H 1965 Proc. Phys. Soc. 851283

Saelee H T 1976 PhD Thesis University of Liverpool

Schlumbohm H 1965 Z. Phys. 182317

Tholl H 1964 Z. Phys. 178183

Wagner K H 1964 Z. Phys. 17864 